\title{
Multiverse Assumptions and Philosophy
}

\author{
James R. Johnson \\ MBA, BS I. E. Retired (Dallas, Texas, USA) \\ E-mail: jrj2222@aol.com
}

\begin{abstract}
Multiverses are predictions based on theories. Focusing on each theory's assumptions is key to evaluating a proposed multiverse. Although accepted theories of particle physics and cosmology contain non-intuitive features, multiverse theories entertain a host of "strange" assumptions classified as metaphysical (outside objective experience, concerned with fundamental nature of reality, ideas that cannot be proven right or wrong) topics such as: infinity, duplicate yous, hypothetical fields, more than three space dimensions, Hilbert space, advanced civilizations, and reality established by mathematical relationships. It is easy to confuse multiverse proposals because many divergent models exist. This overview defines the characteristics of eleven popular multiverse proposals. The characteristics compared are: initial conditions, values of constants, laws of nature, number of space dimensions, number of universes, and fine tuning explanations. Future scientific experiments may validate selected assumptions; but until they do, proposals by philosophers may be as valid as theoretical scientific theories.
\end{abstract}

Keywords: multiverse, metaphysics, philosophy, inflation, string theory, cyclic universe, Loop Quantum Gravity, simulated universe, ultimate universe, fine tuning, infinity

Received 15 January 2017; accepted 20 March 2017

Philosophy and Cosmology, Volume 20, 2018: 8-17

DOI: $10.29202 /$ phil-cosm/20/1

\section{Introduction}

A multiverse (many universes) is a prediction based on a theory or hypothesis. The theory in turn, is predicated on assumptions. However, it is convenient to gloss over the assumptions when contemplating the bizarre ramifications envisioned, such as an infinite number of duplicate yous living in alternate universes. The concept of "duplicate yous living in alternate universes" was described in a now classic article, "Parallel Universes" [Tegmark, 2003]. As science has proven, nature is non-intuitive, even weird at times. For example, in the macro realm, time slows as velocity (or gravity) increases and invisible fields permeate space. Similarly, in the micro world, probabilities decide experimental outcomes and entangled particles are not autonomous. Equally strange concepts or assumptions support speculative theories that predict parallel universes.

(C) Johnson, James R., 2018 


\section{Overview of the Multiverse}

Eleven multiverse proposals are listed in Table 1. Nine of the proposals are similar to those defined by Brian Greene in The Hidden Reality [Greene, 2011, 309]. The proposals represent, on one extreme, an extension of our universe and, on the other extreme, every mathematically possible universe. It could be argued that the Finite Quilted proposal is in a category of its own, since virtually all scientists concur that space, with characteristics identical to our universe, extends beyond what we observe [Tegmark 2014, 126]. Thus, having a limited number of universes in this expanded space establishes the reality of some parallel universes.

As also shown in Table 1, no multiverses have all the same characteristics. Space dimensions range from three to infinity. The Holographic proposal embodies only two universes, while other proposals range from many to infinite. The Cyclic multiverse generates universes parallel in time rather than space. The Simulated and Ultimate proposals allow universes with different laws of physics. Also, eight proposals provide an explanation for fine tuning - a noteworthy characteristic.

Fine tuning is the improbable coincidence that a random collection of about thirty dimensionless input parameters, from the Standard Models of particle physics and cosmology, would produce a universe like ours [Tegmark 2014, 252]. For example, if the cosmological constant were slightly larger or smaller, galaxies could not have formed. If force strengths or particle masses were to vary by a few percent, no atoms, stars or planets would exist - our lifefriendly universe is a rare combination of input parameters. Other parameter permutations might exist in different universes, but the vast majority would be devoid of life.

Physicists have attempted without success to mathematically calculate input parameters values from first principals [Tegmark 2014, 141]. Even string theory physicists have failed at predicting correct values. If these values defy calculation, how do we explain fine tuning? The multiverse provides an answer - if there are billions or trillions or an infinite number of universes, one or more will have the exact constant values we observe. Envisioning an infinite number of universes to prove the existence of one universe seems a bit excessive but, some proponents use this logic as indirect proof of multiverse proposals.

\section{Assumptions for Theories Predicting Multiverse}

"It's not as if physicists are standing ready, multiverse nets in their hand, seeking to snare any passing theory ... into a parallel-universe paradigm. Rather ... proposals emerge unbidden from the mathematics of theories to explain conventional data and observations." This quote documents how independent research efforts directly support the possibility of parallel universes [Greene 2011, 9]. By focusing on these theories, we will identify those key assumptions that defy common sense. The relationships between the key assumptions, theories, and multiverse proposals are shown in Table 2. Because of possible ambiguity between assumptions, resulting calculations, and inherent features, we will assume the word "assumption" has a broad definition. For example, although the nine space dimensions in string theory are classified as assumptions, they might be more accurately defined as inherent features of the theory.

Proposal one. The Finite Quilted multiverse exists within a finite expanse of space, simply more of what we observe, each universe like a patch replicated on a quilt. There may exist hundreds or thousands of Hubble volumes. These parallel universes embody similar initial conditions, the same constants of nature, the same laws of physics, and three space dimensions. The Finite Quilted multiverse adds no insight to fine tuning. 
In the 1920's, Alexander Friedmann showed that a variety of expanding, contracting, or oscillating universes were compatible with Einstein's General Theory of Relativity. At about the same time, George Lemaitre predicted that our universe began with a big bang everything we observe originated in a hot, dense, and rapidly expanding space. Subsequently in the 1940s, as part of the Big Bang theory, Friedmann's student George Gamow calculated that the expanding space, after cooling to about $3000 \mathrm{~K}$, would allow atoms to form and thus free photons to stream through space. The theory predicted that photons from the creation event should be visible in the sky, although at a vastly shifted wave length. When this microwave background radiation was observed in 1964, the theory was validated. The observed universe, sometimes called the Hubble sphere, is the farthest distance we can "see", around 41 billion light years or $4 \times 10^{26}$ meters (the light has been traveling for about 13 billion years, but because space is expanding, the current distance is greater). The Finite Quilted multiverse radius is at least 100 times larger than the Hubble radius based on the observational precision of Cosmic Microwave Background (CMB) data, which did not detect curvature to within a precision of one percent [Tegmark 2014, 127].

The assumptions for the Finite Quilted multiverse are: extended space, finite universe but larger than the observed universe, expanding universe and approximately flat space.

Proposal two. The Infinite Quilted multiverse is similar to the Finite Quilted multiverse except for different initial conditions and the number of parallel universes - instead of thousands of versions, an infinite number of universes populate space and therefore fine tuning can be explained.

Is space finite or infinite? Most multiverses assume an infinite space where reality takes on surprising new meanings - for example, the prediction of duplicate yous (or infinite number of yous) living in an identical world somewhere in another universe. Einstein's equations for general relativity allow for either a finite or an infinite spatial extent. All cosmological models involve an expanding universe, but they differ in the overall shape of space and whether space is finite or infinite [Greene 2011,23]. Although finite space is considered in the inflation framework, infinite space is the favored assumption, which of course permits an infinite number of universes. Also, space is not "nothing" but is governed by laws and contains fields and virtual particles. A brief comment on the implications of "infinity" in the physical world is documented in the Insert below.

Assumptions for the Infinite Quilted multiverse are: space has Infinite extent with no edge and no boundary and space is something.

\section{Insert, Infinity and the Physical World}

An infinite number, when it is reduced by a million or billion or trillions, remains the same: infinite - a truly non-intuitive, incomprehensible notion. There is no experimental evidence of anything infinite in the physical world; infinity is merely a way of speaking; infinite numbers or infinite anything (for example mass, density, and energy, etc.) have no practical meaning. Furthermore, computers do not acknowledge infinity, dealing only with finite digital numbers. Some mathematicians, notably Norman J. Wildberger, also reject the concept, noting, "Mathematics was always about, and always will be about, finite collections, pattern, and algorithms. All those theories, arguments and daydreams involving "infinite sets" need to be recast into a precise finite framework or relegated to philosophy."

Proposal three. In the Inflationary multiverse, the eternal (chaotic) inflation theory produces different constants of nature in separate universes. Sometimes referred to as a 
bubble, each universe is separated by expanding space. Inflation produces an infinite number of universes, and thus provides an explanation for the presence of fine tuning.

Inflationary cosmology is not one unique theory, rather it provides a framework containing many versions differing in details, such as number of inflaton fields (spelled differently than inflation) and their potential-energy curves. However, in general, the basic inflation concept modifies the Big Bang theory by inserting an extremely brief burst of astoundingly rapid expansion during the universe's earliest existence. This stupendous growth explains the uniformity of the $\mathrm{CMB}$ temperature, predicts that the universe is flat, and addresses several other problems associated with the Big Bang.

The source of the brief burst is a tiny speck of space existing in a hypothetical "scalar inflaton field," a field similar to the scalar Higgs field. The inflaton field contains a high amount of potential energy, exists uniformly throughout space, and does not dilute as space expands. The field is subject to random quantum fluctuations that can cause the energy value to vary, and if the energy drops too far, the overall superfast expansion of space stops and inflation starts. In one popular version, the inflation process is astonishingly short, lasting about $10^{-35}$ seconds. During this brief period, space expands by at least $10^{30}$ times. As expansion ends, the field's energy is converted into particles, thus creating a universe. Subsequent expansion was considerably slower (now doubling every 8 billion years) as gravity went to work forming galaxies, stars, and planets.

As proposed by Andrei Linde, eternal or chaotic inflation enhanced Alan Guth's original version by including numerous inflaton fields with random values rather than one field with a constant value. According to eternal inflation, space expands until the scalar field releases both its energy and a uniform negative pressure. This expansion creates a universe with characteristics like our universe. Since eternal implies continuing forever, space must expand faster than it "decays" [Tegmark 2014, 113]. For a mental image, visualize an expanding block of cheese. The holes, sometime referred to as bubbles or pockets, are where inflation stopped; the cheese represents the immense expanse of space surrounding the holes (universes). To appreciate the full scale of this process, consider Brian Greene's re-phasing, "the size of the universe increased by a factor larger than a million trillion trillion in less than a millionth of a trillionth of a trillionth of a second." [Greene 2004, 14]. Now this is real expansion! Inflationary cosmology may be mathematically sound but is incredulous from a human perspective.

Although the brief discussion above assumes that negative pressure in the scalar field creates repulsive gravity (negative or borrowed gravity), this idea makes Max Tegmark nervous, "I just can't shake the uneasy feeling that I'm living a Ponzi scheme of cosmic proportions." [Tegmark 2014, 105]. Thus, even though inflation is based on theoretical physics, such an incredible process leaves room for doubt [Steinhardt, 2011].

Assumptions for Inflationary multiverse are: scalar non-diluting inflaton fields with high potential energy and negative pressure, space doubling its size every $10^{-38}$ seconds, random quantum fluctuations establishing initial conditions, and space expanding faster than it decays.

Proposal four. The Landscape multiverse combines string theory and eternal inflation to predict an infinite number of universes. The significant difference from the Inflationary proposal is that space consists of nine space dimensions instead of the three space dimensions. These extra dimensions provide numerous "shapes" for space, each shape a different variety of universe - more than enough to explain fine tuning.

In the first version (1984) of superstring theory, now referred to as string theory, tiny strings or vibrating filaments, replace electrons and quarks as nature's building blocks. 
Strings are so minute they may never be observed (Planck distance of $10^{-33} \mathrm{~cm}$ ). The string vibration pattern dictates intrinsic features that may represent an electron or a quark or more importantly a graviton (massless, chargeless, and having a spin-2 quantum property). Thus, without contradicting previous theories, string theory bridged the gap between general relativity and quantum mechanics. However, the mathematics, as defined in five unique theories, required nine rather than three dimensions for space. The extra dimensions are curled up into Calabi-Yau shapes, shapes that dictate particle properties. Unfortunately, there are "many" possible shapes $\left(10^{500}\right)$ for the extra dimensions and no way to select the one that matches existing particles [Greene 2011, 126]. If the multiverse exists, then all options may be realized.

Assumptions for the Landscape multiverse are: tiny vibrating strings, nine dimensions of space and one string vibration pattern for graviton.

Proposal five. The Brane multiverse evolves from the current version of string theory, which has ten space dimensions. The theory predicts multi-dimensional branes (explained below). In this environment, our universe might exist on a three-dimensional brane along with possibly an infinite number of similar branes. If so, fine tuning is explained.

In 1995, physicist Ed Witten started the second revolution of string theory by employing refined calculations. He showed that the five previously unique string theories were encompassed in one overriding theory, M-theory. The old calculations missed one dimension; there were actually ten space dimensions. M-theory generates " $n$-dimensional" braneworlds, where $\mathrm{n}$ has values from one to nine. A "one" brane corresponds to a onedimensional string and a "three" brane corresponds to a three-dimensional space. Thus, our universe could exist on a 3-brane (one of many) with large or infinite extent. Different branes reside in different dimensions, not necessarily separated by vast distances in space, and possibly hovering in close proximity to each other. All strings are attached to the brane except for gravitons, which are unattached loops that can leave and re-enter the 3-brane [Greene 2011, 118].

Assumptions for the Brane multiverse are: ten space dimensions, branes with one to nine space dimensions, strings attached to branes except for graviton strings, and the observed universe resides on a 3-brane.

Proposal six. The Cyclic multiverse is another prediction based on M-theory. In a process that repeats over time, two colliding branes generate new universes. Although laws remain unchanged, the constants of nature may vary as the process repeats. Consequently, there is an explanation for fine tuning.

Some theorists predict periodic collisions between braneworlds that produce Big Bang scenarios. The proposed cycle, occurring over a trillion years, is: collision, expansion, cooling, dispersion and then another collision. Although collisions occur in long cycles, infinite time generates an infinite number of universes that repeat in time. The process excludes a dramatic "inflation" type of expansion. This theory not only addresses the finetuning issue but also the "infinite regress enigma" by answering the question, what does the last turtle holding up the universe stand on? Since the cycle is continuous, it needs no turtles.

Assumptions for the Cyclic multiverse are: branes physically collide in trillion-year periods and, brane collisions produce Big Bang-type events (inflation's dramatic expansion not required).

Proposal seven. In the bounce multiverse theory, expansion is created from the rebound of a contracting universe (an alternative to inflation). It is based on Loop Quantum Gravity (LQG). Fine tuning is explained. 
LQG predicts the existence of spacetime atoms each with a volume of $10^{-99} \mathrm{~cm}^{3}$. Particles and fields are described as spin networks. Spacetime is spin foam with discrete time. Loop quantum gravity suggests that the atomic structure of spacetime changes the nature of gravity at very high energy densities. Loop-based scenarios are founded on general principles of quantum theory and relativity theory and therefore avoid introducing new ad hoc assumptions (as with inflation). When a preexisting universe collapses under the attractive force of gravity, the density grows so high that gravity switches to repulsive and the universe starts expanding again. It bounces [Bojowald, 2008]. In this scenario energy is recycled from the previous universe.

Assumptions for LQG are: spacetime atoms, repulsive gravity, bounce from previous universe.

Proposal eight. The Holographic multiverse represents two physically equivalent universes, one existing on a two-dimensional boundary surface mirroring a second universe in three-dimensional space. Fine tuning is not resolved.

From black-hole theories, physicists conclude that the amount of information contained within a region of space is always less than the area of the surface that surrounds the region [expressed in square Planck units), a strange relationship, since volume increases with the cube of a sphere's radius. Consequently, since all physical phenomena can be encoded on the surrounding surface, our three-dimensional reality could be a holographic projection from a two-dimensional surface - the holographic principle. Brian Greene views this development as among the most exciting in decades: "A particular nongravitational, point particle quantum field theory in four spacetime dimensions describes the same physics as strings, including gravity, moving through a particular swath of ten spacetime dimensions" [Greene 2011, 266]. Restated, string and quantum field theories are equivalent approaches expressed in different languages. Others have proposed holographic models reinforcing the concept [Afshordi et al., 2014].

The Holographic multiverse assumptions are: holographic math (string theory and quantum field theory), and a two-dimensional surface projects a three-dimensional reality.

Proposal nine. In the Quantum multiverse, an apparent wave function collapse spawns a parallel universe in Hilbert space (abstract space where the wave functions live). Rather than a wave collapsing abruptly when "observed," it continues in a separate, previously identical universe. No insight into fine tuning is provided.

As Yogi Berra and physicist Hugh Everett said, "When you come to a fork in the road, take it." In our context, does the wave function, expressed mathematically by the Schrodinger equation, collapse as the Copenhagen interpretation predicts or are all probabilities realized in separate worlds as in the Many Worlds interpretation? There is no consensus on the answer to this question. Where quantum physics is governed by the Copenhagen interpretation, the wave function collapses into one outcome - no other outcome occurs. On the other hand, where quantum physics is governed by the Many Worlds interpretation, each quantum fork in the road splits the universe in Hilbert space (abstract infinitely dimensional space). Since quantum decisions are prevalent, the process naturally generates many parallel universes.

Assumptions for Quantum multiverse are: non-collapsing wave function (Many Worlds interpretation) and Hilbert theoretical space with possibly infinite dimensions.

Proposal ten. In the Simulated multiverse, we live in a computer simulation. This option is significantly different from the preceding proposals, since a "computer program" may modify the basic laws of nature. Any number of simulations could exist so the simulated environment explains fine tuning. 
The key underlying assumption is based on sentience, the ability to create conscious self-aware beings within a supercomputer. Is this possible? Science fiction writers have often envisioned integrating people into a simulated reality as in the movie The Matrix. However, others do not think it is possible; they believe life is more than physical atoms; life requires a non-physical component (spirit or soul) that is non-programmable. However, it may be possible for an inquisitive and advanced civilization possessing powerful computers to simulate virtual universes. If we exist in their simulation, hopefully, they do not lose interest and terminate the simulation any time soon.

Our computers operate on computable functions and discrete numbers that would necessitate a digital simulation [Moyer, 2012]. If nature is continuous, a new, distinctive type of supercomputer must be employed, one capable of handling numbers with an infinite number of digits, such as, $\pi$ or 2 (or possibly a new form of mathematics to express these numbers as speculated by Norman Wildberger).

The Simulated multiverse assumption are: ability to simulate self-aware beings, an advanced civilization with motivation to experiment, and discrete computations or unique supercomputers.

Proposal eleven. The Ultimate multiverse is appropriately labeled since it assumes all mathematically possible universes exist. Thus, it encompasses all the above proposals plus others based on more fundamental and possibly different laws - in this context, different laws than we experience. All characteristics can assume any value; thus, fine tuning is explained.

The key hypotheses predicting parallel universes are the External Reality Hypothesis (ERH) and the Mathematical Universe Hypothesis (MUH). As implied from their names, all reality is described mathematically; in fact, physical existence equals mathematical relationships and all defined mathematical relationships are real. Since every imaginable universe may not have a mathematical definition, some would not exist. If there are relationships in nature based on continuous numerical values (infinite digits are needed to define continuous values) or endless computations, the MUH has a problem since non-computable relationships could never become reality. One solution is to allow only computable functions as Max Tegmark acknowledges, "computable functions may be needed for MUH to make sense" [Tegmark 2014, 357].

In the MUH, the passage of time is not fundamental - a time-dependent process is not required, the flow of time is an illusion [Davies, 2002]. Thus, existence is like a movie DVD that just exists, not physically but as mathematical equations [Tegmark 2014, 318]. A baking analogy underscores the concept of MUH - the mathematical relationships exist just as the recipe for a cake exists. According to the hypothesis, because the recipe contains the relationships, it is as real as the physical cake.

Assumption for Ultimate multiverse are: mathematic relationships define reality (MUH), relationships exist outside space and time, and time is not fundamental.

\section{Future Discoveries}

There are scientific experiments that may validate key assumptions. For example, during exponential expansion (inflation), tiny quantum fluctuations in the gravitational field are stretched into long-wavelength gravitational waves. It is predicted that these waves would polarize CMB photons in a pinwheel pattern. Thus, confirming polarization of CMB photons directly supports inflation [Krauss, 2014]. Also, more precise CMB data might validate the reasonableness of existing scalar field potential energy curves. Conversely, the legitimacy of the fundamental inflation theory is challenged by competing theories. 
There are also several experiments attempting to discover extra dimensions and, if successful, as Brian Greene says, “...would so thoroughly shake the foundations of physics ... that we must be willing to question basic, seemingly self-evident, elements of reality." [Greene 2011, 426]. The Large Hadron Collider (LHC) experiments address extra dimensions in two ways. First, if energy is lost in LHC experiments, it may be escaping to other dimensions. And second, at short distances, gravity may have greater strength (not flow into other dimensions), allowing mini black holes to form more easily. Since mini black holes should leave a unique signature when disintegrating, experimental data from LHC could validate their existence, inferring that at greater distances gravity does leak into other dimensions. Related to this last experiment, if extra dimensions are as "large" as $10^{-14}$ meters, the sensitive cosmic-ray telescope, Pierre Auger Observatory in Argentina, may detect debris from mini black holes created when cosmic rays hit earth's atmosphere. Additionally, if in other experiments, scientists were to measure a stronger force for gravity at microscopic distances (say from $10^{-5}$ to $10^{-6}$ meters), it would corroborate the idea that the force is spreading to another dimension.

Next addressing strings, a key aspect of string theory is supersymmetry that predicts a heavier partner particle for each existing particle in the Standard Model. If the LHC identifies the matching new particles, then an important part of string theory would be validated. Since the M-theory braneworld string vibration energies are considerably weaker than the conventional string energies, it may be feasible for the LHC to detect new particles corresponding to expected vibration patterns.

The cyclic process, relying on brane collisions, might produce gravitational waves, but because there is no inflationary stretching, there should be no evidence of gravitational waves in $\mathrm{CMB}$ data. If detected, they would invalidate the predicted Cyclic multiverse. Also, were accelerated expansion of the universe to stop, the cyclic concept would be disproved because it predicts continuous expansion.

In the bounce scenario, gravitational waves might have made an imprint on the cosmic microwave background radiation bringing information from a time near to, or even before, the big bang [Bojowald, 2008].

\section{Criteria, Evidence}

This article focused on those assumptions that ultimately predict a multiverse. We traced the path from multiverse proposals to supporting theories to fundamental assumptions. A knowledgeable debate must focus on those assumptions. Some assumptions, although inherent in leading scientific theories, appear quite strange. The Quilted Finite parallel universe, with a limited number of universes, establishes a safe baseline; but opinions on the other ten multiverse proposals are based on the validity of the assumptions. Since many assumptions are non-intuitive, challenging their credibility is expected [Ellis, 2011].

On the other hand, is intuition an appropriate measure? Nature is weird, relativity and quantum mechanics both contradict intuition - "and should (we) expect the ultimate theory of physics, whatever it turns out to be, to feel weirder still?" [Tegmark 2014, 363]. From this perspective, radical new ideas are anticipated.

Since parallel universes reside forever outside our vision, future experiments and theoretical calculations can provide only circumstantial evidence for their existence. If experiments are not conclusive, then we are in the realm of metaphysics where proposals by philosophers are as valid as theoretical scientific theories. 


\begin{tabular}{|c|c|c|c|c|c|c|c|}
\hline & $\begin{array}{l}\text { Multiverse } \\
\text { Proposals }\end{array}$ & $\begin{array}{c}\text { Different } \\
\text { Initial } \\
\text { Conditions }\end{array}$ & $\begin{array}{l}\text { Different } \\
\text { Constants }\end{array}$ & $\begin{array}{c}\text { Different } \\
\text { Laws }\end{array}$ & $\begin{array}{c}\text { Number } \\
\text { of } \\
\text { Dimen- } \\
\text { sions }\end{array}$ & $\begin{array}{c}\begin{array}{c}\text { Number } \\
\text { of }\end{array} \\
\text { Universe's }\end{array}$ & $\begin{array}{c}\text { Explains } \\
\text { Fine } \\
\text { Tuning }\end{array}$ \\
\hline 1 & Quilted, Finite & No & No & No & 3 & 1,000 's & No \\
\hline 2 & $\begin{array}{l}\text { Quilted, } \\
\text { Infinite }\end{array}$ & Yes & No & No & 3 & $\infty$ & Yes \\
\hline 3 & Inflationary & Yes & Yes & No & 3 & $\infty$ & Yes \\
\hline 4 & Landscape & Yes & Yes & No & 9 & $\infty$ & Yes \\
\hline 5 & Brane & Yes & Yes & No & 10 & $\infty ?$ & Yes \\
\hline 6 & Cyclic & Yes & Yes & No & 10 & $1, \infty$ & Yes \\
\hline 7 & Bounce & Yes & Yes & No & 3 & $1, \infty$ & Yes \\
\hline 8 & Holographic & No & No & No & 10 & 2 & No \\
\hline 9 & Quantum & No & No & No & $\infty$ ? & $<_{\infty}$ & No \\
\hline 10 & Simulated & Yes & Yes & Yes & 3 & Many & Yes \\
\hline 11 & Ultimate & $\mathrm{n} / \mathrm{a}$ & Yes & Yes & Many & $\infty$ & Yes \\
\hline
\end{tabular}

Definitions of the characteristics are: initial conditions - how things started, the general distribution of mass which forms galaxies and galaxy clusters on a vast scale; constants of nature - the masses and forces calculated from about thirty dimensionless input parameters within the Standard Model of Particle Physics and the Standard Model of Cosmology; laws - the laws of physics represented by equations (Einstein's special and general relativity, Maxwell's electromagnetism, Schrodinger's quantum mechanics, and Friedmann's expanding universe); number of space dimension - typically as observed in our universe, three space dimensions (and one of time); number of universes - count of other universes existing in space; and lastly fine tuning - specific values for the constants of nature allowing elements, planets, stars, galaxies, and life, specifically us, to exist

Table 1. Characteristics of parallel universes

Multiverse Theory

Quilted, General Relativity, Finite Finite Space

2 Quilted, General Relativity, Infinite Infinite Space

3 Inflationary

Eternal Inflation

4 Landscape

5 Brane

6 Cyclic

7 Bounce
String Theory, Eternal Inflation

String Theory, Branes

String Theory, Collisions

Loop Quantum Gravity

\section{Key Assumptions}

Extended Space

Infinite Space

Scalar Inflaton Field, Exponential Expansion

Strings, Nine Dimensions, Scalar Inflaton Field

Strings, Ten Dimensions, Branes

Strings, Ten Dimensions, Branes, Collisions

Spacetime Atoms, Repulsive Gravity 
8 Holographic String Theory, Holographic

Holographic Math (Branes, Quantum Field Theory)

9 Quantum Quantum Mechanics

Non-Collapsing Waves in Hilbert Space

10 Simulated Sentience Hypothesis

Self-Aware Supercomputers, Advanced

Civilization

11 Ultimate

Mathematical Universe Hypothesis

Mathematical Relationships Define Reality

Table 2. Theory and key assumptions for multiverse proposals

\section{[Dd References}

Afshordi, N., Mann, R. and Pourhasan, P. The Black Hole at the Beginning of Time. Scientific American, August, 311(2), 2014: 37-43.

Bojowald, M. Follow the Bouncing Universe, Scientific American, October, 299(4), 2008: 44-51.

Davies, P. That Mysterious Flow. Scientific American, February, 287(3), 2002: 40-47.

Ellis, G. Does the Multiverse Really Exist? Scientific American, August, 305(2), 2011: 38-43.

Greene, B. 2004. The Fabric of the Cosmos. Alfred A. Knopf, New York. ISBN-10: 0-37541288-3.

Greene, B. 2011. The Hidden Reality. Alfred A. Knopf, New York. ISBN-10: 0-307-26563-0.

Krauss, L. A Beacon from the Big Bang, Scientific American, October, 305(4), 2014: 59-67.

Moyer, M. Is Space Digital? Scientific American, January, 308(1s), 2012: 104-111.

Steinhardt, P. The Inflation Debate, Scientific American, April, 304(4), 2011: 36-43.

Tegmark, M. Parallel Universes, Scientific American, May, 288(5), 2003: 40-51.

Tegmark, M. Our Mathematical Universe: My Quest for the Ultimate Nature of Reality. 1st Edh., Knopf Doubleday Publishing Group, New York, 2014. ISBN-10: 0-307-59980-3. 